\title{
Salivary exosomal microRNAs as biomarkers for head and neck cancer detection-a literature review
}

\author{
Cosmin Ioan Faur ${ }^{1}$, Horatiu Rotaru ${ }^{2 *}$ (D), Ciprian Osan ${ }^{3}$, Ancuta Jurj ${ }^{4}$, Rares Calin Roman², Madalina Moldovan², \\ Magdalena Chirila ${ }^{5}$ and Mihaela Hedesiu ${ }^{1}$
}

\begin{abstract}
Background: MicroRNAs (miRs) are small, non-coding mRNA molecules which regulate cellular processes in tumorigenesis. miRs were discovered in extracellular environment and biological fluids, carrying marks of head and neck squamous cell carcinoma (HNSCC). They were also identified in abundance in salivary exosomes, in which they are protected by exosome lipid barrier against enzymatic injuries and therefore, the accuracy of exosomal miR-based cancer detection increase. This systematic review aimed to reveal and inventorize the most reliable exosomal miRNAs in saliva samples which can be used as novel biomarkers for early detection of HNSCC.
\end{abstract}

Materials and methods: A systematic literature search, according to PRISMA guideline, was performed on Pubmed and Google Academic libraries, based on specific keywords. Original articles published between 2010 and 2021 were selected. The quality of each paper was assessed using the Quality Evaluation Scoring Tool.

Results: At the end of selection process, five studies met the inclusion criteria. These studies analyzed twelve salivary exosomal miRs, presenting different methods of exosome and miR identification for HNSCC detection. A comprehensive explanation of the miR pathways of action was drawn and illustrated in this review.

Conclusion: Exosomal miRs are promising biomarkers for oral cavity and oropharyngeal cancer detection. miR-10b-5p, miR486-5p, miR-24-3p and miR-200a stand as the most useful ones in saliva sample examination.

Keywords: HNSCC, Exosome, Extracellular microRNA, Biomarker, Saliva

\section{Background}

Affecting a large variety of anatomic subsites such as oral cavity, pharynx, larynx, salivary glands, nasal fossa and paranasal sinuses, early and non-invasive head and neck cancer detection is a challenging topic [1]. The most frequent outset origin is from squamous cells of the epithelium, but the anarchic proliferation of mesenchymal or neural cells could also be involved. The annual incidence of head and neck squamous cell carcinoma (HNSCC) is more than 900,000 new cases worldwide, and it is greatly correlated with tobacco smoking and chewing, alcohol consumption and HPV

\footnotetext{
* Correspondence: dr.horatiu.rotar@gmail.com

2Department of Oral and Cranio-Maxillofacial Surgery, "Iuliu Hatieganu"

University of Medicine and Pharmacy, 400033 Cluj-Napoca, Romania

Full list of author information is available at the end of the article
}

infection [2,3]. Despite the considerable diversification of therapeutic methods, long-term survival rate remains under $50 \%$ due to the late diagnosis, frequent onset of multiple primary tumors and tumor relapse [4]. At initial presentation, more than $40 \%$ of patients are found with regional nodal involvement (HNSCC stage IVA or B), and 10\% are diagnosed with distant metastases (HNSCC stage IVC), correlated with a poor prognostic [5]. Therefore, the interest in discovering new methods for early cancer detection is very high, promoting immediate therapy to increase patient overall survival rate and quality of life.

The exosomes are 50-150-nm extracellular vesicles (EV) involved in the intercellular communication. Exosome biogenesis is a complex process which involves 3 main parts: endosome formation by the inward budding 
of the plasmatic membrane, the generation of multivesicular bodies (MVB) containing intraluminal vesicles and the fusion between MVBs and cellular membrane, resulting in the release of the exosomes in the extracellular environment [6]. The structure of this EV is related to the original cell, being composed of a lipidic double layer that surrounds the internal components: proteins, lipids, DNA, RNA and miRs. In addition, exosomes possess specific surface proteins that can be used for differentiation between these nanoparticles and other microvesicles or apoptotic bodies [7]. These membrane proteins, such as tetraspanin CD81 or CD9 allow sorting, selective recruitment and profiling of the cancer cell-derived exosomes [8].

MicroRNAs (miRs) are 22-nucleotide small noncoding RNAs that interact with the RNA-induced silencing complex (RISC), binding to the 3' untranslated region (UTR) of mRNA to induce either mRNA degradation or mRNA translation inhibition into specific proteins [9]. Regulating the target gene expression, miRs influence vital processes including cell cycle, apoptosis, proliferation and differentiation. Moreover, any dysregulation of miR expression may contribute to the outset, development and invasion of various types of cancer, including HNSCC [10]. Besides the cytoplasmatic miRs, a considerable number of scientific research highlighted the ability of this molecule to survive in extracellular environment. According to recent studies, there are 4 primordial mechanisms of miR secretion into extracellular space: through exosomes, shedding vesicles, apoptotic bodies and in association with proteins or high-density lipoproteins [11]. Being packed into exosomes, miRs are protected from enzymatic degradation against endogenous RNase. As a result, they are more stable in the extracellular environment compared with cell-free miRs. Exosomal miRs can be identified in body fluids, such as blood and saliva, making them optimal candidate biomarkers for malignant tumors. Several miRs, such as miR-3714, miR-650 and miR-575, were identified significantly altered in the saliva of nasopharyngeal carcinoma patients comparing with healthy controls, which emphasize the opportunity of using salivary miRs as a method of diagnosis [12].

Despite of the abundance of cancer biomarkers identified recently in saliva, none of them was certified as an indisputable fingerprint of malignancy. Moreover, exosome isolation and characterization, as well as exosomal miR expression quantification, have various methods of investigation which could induce heterogeneous results.

The purpose of this review is to spread light over this biomarker-based diagnostic method and to identify the most reliable exosomal microRNAs in saliva as an early, effective, and non-invasive diagnosis tool for HNSCC.

\section{Materials and method Publication search}

A search on Pubmed and Google Academic databases was performed according to PRISMA methodology 2015 Checklist algorithm for systematic reviews and metaanalysis [13]. The strategy for literature search was represented by "((saliva and exosome) or (saliva and microRNA) or (exosome and microRNA)) and (head and neck cancer)" on Pubmed.gov and "salivary exosomal microRNA" on Google Academic.

\section{Inclusion and exclusion criteria}

A year publication filter was applied for 2010-2021 interval of time. The studies selected from both databases were original articles only. An original article was defined as a research performed on clinical subjects or a laboratory research which presents original data. Reviews and metaanalysis were excluded. Duplicated papers were also removed. The abstract and full text were further analyzed. The original articles which did not focus on the topic of head and neck cancer detection or premalignant lesion detection using salivary exosomal miR as biomarkers were excluded. In the following stage, the eligibility criteria of the studies were applied, as listed below:

(1) Availability of the text in full format;

(2) Head and neck cancer or premalignant lesion detection or management;

(3) Saliva samples from patients and control as material for research;

(4) Salivary exosomal microRNA;

(5) Quantification of the parameters of test in terms of sensitivity, specificity, AUC or ROC;

(6) Results presented as quantitative data;

(7) Article written in English;

(8) Respecting the structure of IMRAD (introduction, material and method, results, discussions).

Each article that did not respect all of the criteria was excluded from the full-text analysis.

\section{Qualitative assessment}

The QUEST quality assessment tool was used to evaluate all studies included in this systematic review. This tool comprises of 6 quality parameters: Authorship, Attribution, Conflict of interest, Currency, Complementarity and Tone. Each article was scored from 0 to 28 according to the above-mentioned parameters, a higher value indicating a reliable methodology. To diminish the risk of bias, two distinct reviewers analyzed the selected articles using the Quality Assessment of Diagnostic Accuracy Studies Tool (QUADAS-2), including four main domains: patient selection, index test, reference standard, flow and timing [14]. 


\section{Results and discussion}

A total of 700 articles (687 on Pubmed.gov and 23 on Google Academic) resulted in the searching process. Two articles were duplicated and therefore excluded. A number of sixty-four original papers were selected for the screening stage of the abstract, the rest of the articles being excluded after title examination. Following the screening process, fifty-nine articles were removed from the analysis due to not meeting all of the aforementioned criteria. As a result, five articles were included for full-text analysis and review (Fig. 1), four focused on cancer and one on premalignant lesion detection (Table 1).

The study design was different between papers (Table $1)$. While three articles focused only on the examination of saliva samples, the other two included in vitro analysis of cell culture, among which, one specific research paper performed a supplementary examination on tissue sample. The studies including more analysis than saliva samples better explained the influence of miRs in head and neck cancer (HNC).

\section{Quality indicators}

The articles were qualitatively assessed through QUEST review analysis. All papers obtained a strong qualificative after analysis, greater than 26, that classify them as highquality studies. In addition, after QUADAS- 2 analysis, all five articles presented a low bias risk and high applicability in the four parts of the test (Table 2).

\section{Saliva samples}

Saliva is named by some authors "body mirror" due to the large amount of fingerprints of systemic diseases or tumors [20-22]. Besides exosomal miRs, other fingerprints are also present in the saliva. Nucleic acids (e.g. cell-free miR (cfmiR), mRNA, various types of DNA), proteins, cytokines (e.g. Il-8, Il-6, Il-10), circulant tumor cells (CTC) or other extracellular vesicles are examples

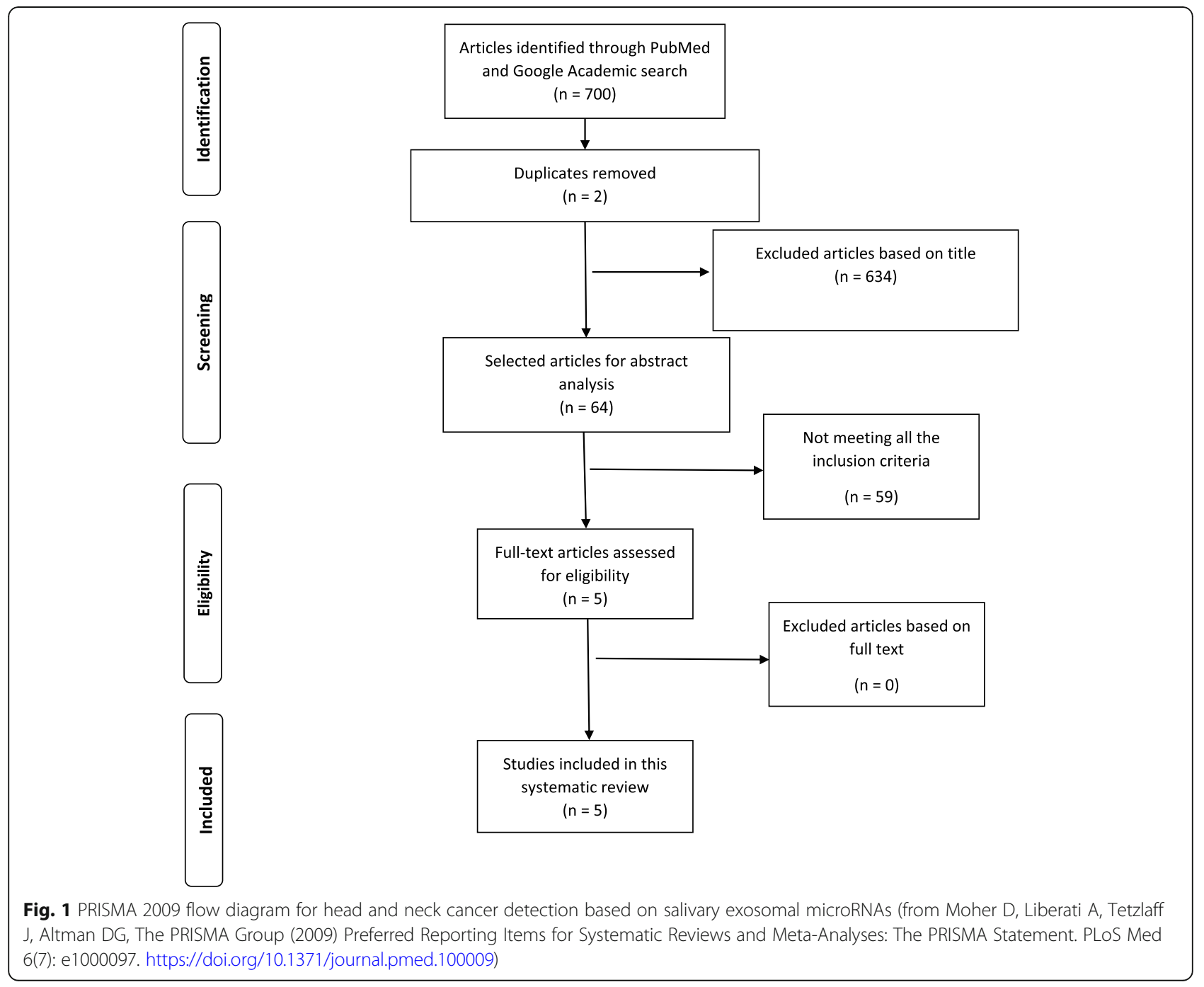


Table 1 Articles included for this systematic review and quantity of saliva required for exosomal miR detection

\begin{tabular}{|c|c|c|c|}
\hline $\begin{array}{l}\text { Author and } \\
\text { publication year }\end{array}$ & Title & Study type & $\begin{array}{l}\text { Saliva } \\
\text { sample } \\
\text { (ml) }\end{array}$ \\
\hline $\begin{array}{l}\text { Byun et al. } 2015 \\
{[15]}\end{array}$ & Diagnostic profiling of salivary exosomal microRNAs in oral lichen planus patients & Saliva & 5 \\
\hline $\begin{array}{l}\text { Langevin et al. } \\
2017[16]\end{array}$ & $\begin{array}{l}\text { Comprehensive microRNA-sequencing of exosomes derived from head and neck carcinoma } \\
\text { cells in vitro reveals common secretion profiles and potential utility as salivary biomarkers }\end{array}$ & Saliva and in vitro & 2 \\
\hline Gai et al. 2018 [17] & $\begin{array}{l}\text { Salivary extracellular vesicle-associated miRNAs as potential biomarkers in oral squamous cell } \\
\text { carcinoma }\end{array}$ & Saliva & - \\
\hline He et al. 2020 [18] & $\begin{array}{l}\text { Salivary exosomal miR-24-3p serves as a potential detective biomarker for oral squamous cell } \\
\text { carcinoma screening }\end{array}$ & $\begin{array}{l}\text { Saliva, in vitro and } \\
\text { tumor tissue }\end{array}$ & 5 \\
\hline $\begin{array}{l}\text { Farag et al. } 2021 \\
{[19]}\end{array}$ & $\begin{array}{l}\text { MiR-134/miR-200a-derived salivary exosomes are novel diagnostic biomarkers of oral } \\
\text { squamous cell carcinoma }\end{array}$ & Saliva & 3 \\
\hline
\end{tabular}

"_" = unspecified

of salivary biomarkers of tumors or systemic diseases $[23,24]$.

The presence of various biomarkers in saliva makes it relevant for liquid biopsy examination [25]. Moreover, saliva is a friendlier environment for various biomarkers. For example, the lower quantity of ribonuclease compared with blood reduces the degradation of miRs [26, 27]. Saliva is a cost-effective sample for cancer detection while the harvesting is easy and non-invasive, does not require trained personnel and transport, and deposit is less sensitive than for other types of samples [28, 29].

Exosomal miRs, like other biomarkers of cancer, are released by the tumor cells in the peritumoral environment, and they are further driven either in the blood stream or in the tumor lavage fluids, such as saliva [30, 31]. However, the salivary exosomal cargo originates from the lavage process and blood stream from where exosomes passively pass into saliva through gingival sulcus fluid. As a consequence, the concentration of exosomal miRs in saliva is higher than in blood [32].

The size of salivary samples used for exosomal miR identification varied in the papers reviewed, from 2 to 5 $\mathrm{ml}$ (Table 1) [15-19]. Still, Gallo et al. obtained exosomal miR from $1 \mathrm{ml}$ of saliva, which proves that a small amount of saliva sample contains sufficient exosomal $\mathrm{miR}$ for a proper analysis [33].

\section{Isolation and characterization of Exosomes}

Exosomal miR analysis from saliva samples requires isolation and characterization of the exosomes and identification of miRs and quantification of their expression (Fig. 2). In the studies selected for this review, exosomes were isolated by ultracentrifugation ( 2 articles), by using extraction kits ( 2 articles) or by filters ( 1 article) (Table 3). Langevin et al. (2017), as well as Farag et al. (2021), used differential ultracentrifugation due to the bias that can appear in commercial affinitybased approaches (e.g. extraction kits) for isolation of cancerassociated exosomes [16, 19]. Differential ultracentrifugation is the golden standard for exosome isolation, and it consists of removal of viable cells, cellular debris and macromolecular proteins by centrifugation at different speeds, ended by 100 , $000 \times$ g ultracentrifugation for 70 minutes to obtain the exosomes. This method is time-consuming, labor-intensive, and instrument-dependent, associated with a risk of exosome losing [25, 34]. He et al. (2020) and Byun et al. (2015) used a kit for exosome purification (ExoQuick-TCTM -SBI, Mountain View, CA, USA) and a protocol of centrifugation with reduced $g$ force (1500), that is stated by the authors to be quicker, easier and more feasible for cancer screening [15, 18]. In contrast with the above-mentioned methods, Gai et al. (2018) used filters of $0.2 \mu \mathrm{m}$ and $1500 \times$ g protocol of centrifugation, which is also a short-time technique [17]. Even though all of the aforementioned protocols lead to exosome

Table 2 QUADAS-2 assessment of the risk bias and applicability for the included articles in this review

\begin{tabular}{|c|c|c|c|c|c|c|c|}
\hline \multirow[t]{2}{*}{ Study } & \multicolumn{4}{|l|}{ Risk of bias } & \multicolumn{3}{|c|}{ Applicability concerns } \\
\hline & Patient selection & Index test & Reference standard & Flow and timing & Patient selection & Index test & Reference standard \\
\hline Byun et al. [15] & $?$ & + & + & + & + & + & + \\
\hline Langevin et al. [16] & + & + & + & + & + & + & + \\
\hline Gai et al. [17] & + & $?$ & + & + & + & + & + \\
\hline He et al. [18] & + & + & + & + & + & + & + \\
\hline Farag et al. [19] & + & + & + & + & + & + & + \\
\hline
\end{tabular}

+ Low risk; - High Risk; ? Unclear 


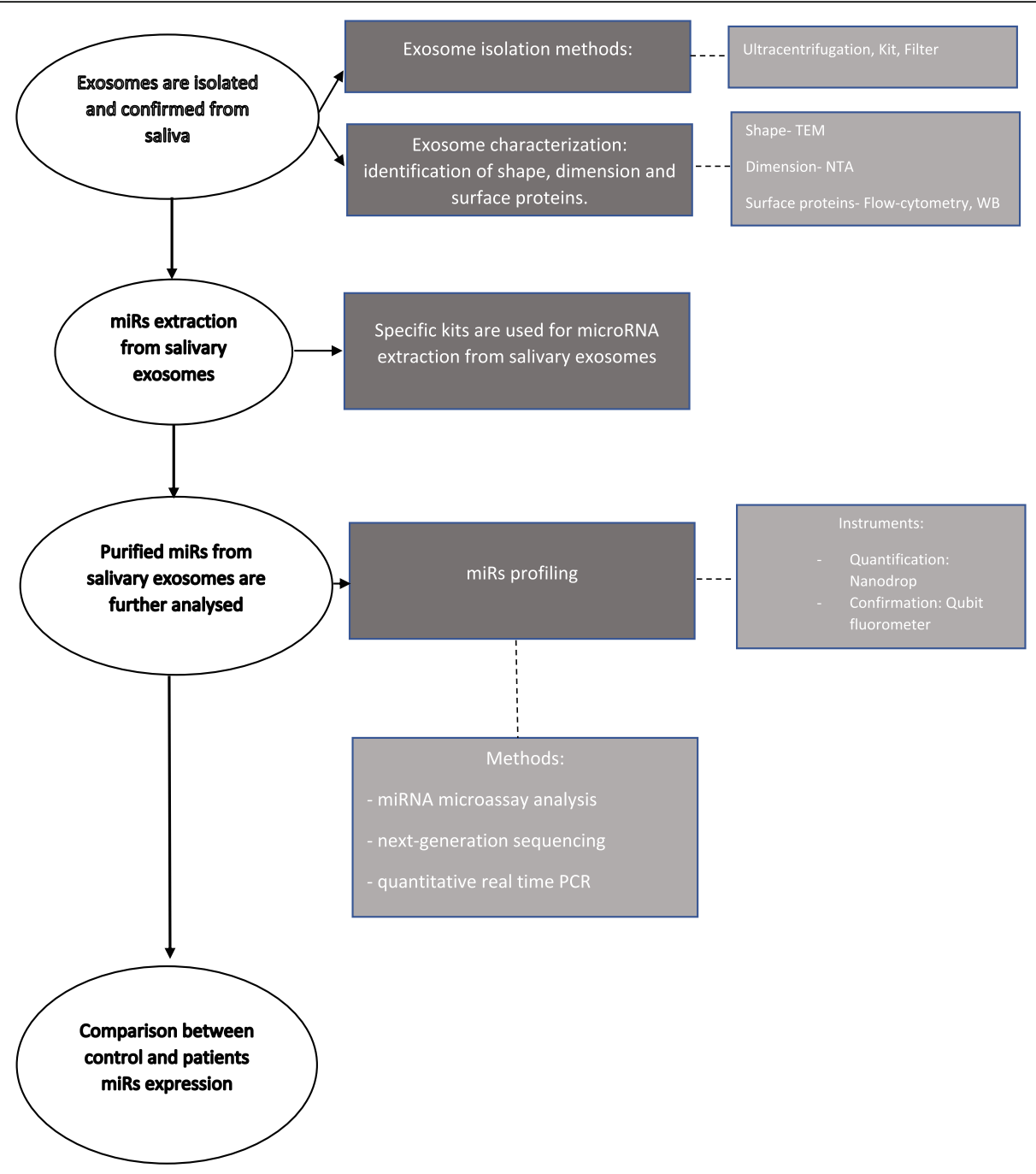

Fig. 2 miRs' extraction methods and quantification of their expression from salivary exosomes of the control and cancer patients

isolation and purification, different types of exosome isolation kits offer different amounts of exosomes and different dispersion stability. Therefore, these variations reduce reproducibility between measurements and cause inconsistency when it comes to result comparison from different laboratories [35, 36]. Exosome purification is a sensitive process and the results may be influenced by debris, bacterial flora and various types of sample harvesting (e.g. stimulated or unstimulated saliva) [25].
Characterization of the exosomes defines the physicochemical properties of exosomes, such as size, shape, surface charge, density and porosity [35]. In the papers reviewed, transmission electron microscopy (TEM), nanoparticle-tracking analysis (NTA), negative staining, flow cytometry and western blot (WB) were used for the confirmation stage of analysis. TEM was used in all of the articles included for this study. In addition to TEM, Byun et al. (2015) used flow cytometry by Exo-Flow kits

Table 3 Isolation and characterization of exosomes

\begin{tabular}{lll}
\hline Author & Exosome isolation & Exosome characterization \\
\hline Langevin et al. (2017) [16] & Ultracentrifugation & TEM, NTA, WB (CD 81, TSG 101) \\
Farag et al. (2021) [19] & Ultracentrifugation & TEM \\
Byun et al. (2015) [15] & Kit & TEM, Flow cytometry \\
He et al. (2020) [18] & kit & TEM, NTA, negative staining, WB (CD81, CD63, TSG101) \\
Gai et al. (2018) [17] & filters & TEM, NTA, negative staining, WB (CD9, CD63, TSG101) \\
\hline
\end{tabular}


(SBI) for the confirmation of the exosomes [15]. Langevin et al. (2017) used TEM for visual confirmation, WB for immunohistochemical confirmation and NTA for quantification of exosomes [16]. Additionally, $\mathrm{He}$ et al. (2020) and Gai et al. (2018) included negative staining to improve the characterization accuracy $[17,18]$. NTA is a biophysical approach used for optical particle tracking performed for exosome characterization, that can measure the concentration and size distribution of vesicles in the 10 to $1000-\mathrm{nm}$ range [35]. TEM is also a biophysical method that uses large objectives on microscopes, in a special and controlled environment, for describing the size, shape and aspect of the exosomes. For example, exosomes can be seen on TEM examination as 30-150$\mathrm{nm}$ microvesicles, rounded or irregular, alone or clustered [35]. Farag et al. (2021) described exosomes as a heterogeneous population in oral squamous cell carcinoma (OSCC) and in tobacco smoking patients, presenting irregular morphology and various shapes and dimension (ranging from 30 to $400 \mathrm{~nm}$ ) [19]. On the contrary, exosomes observed in healthy subjects' saliva revealed homogeneous circular microvesicles with a size ranging from 60 to $90 \mathrm{~nm}$. Additionally, large vesicle aggregates were observed in the OSCC samples compared with smokers' samples [19]. These findings regarding exosome characterization were in accordance with Gai and Sharma's results $[17,37]$. Conversely, saliva of patients who suffer from premalignant lesions, such as oral lichen planus (OLP), proved to contain typical rounded vesicle structures sized 20 to $100 \mathrm{~nm}$, more similar to healthy patients' saliva [19]. Moreover, a large concentration of exosomes was discovered in cancer patients' saliva compared with healthy subjects' [19]. The explanation of the increased number of exosomes and the change in shape may be the tumor-associated hypoxia $[26,38]$.

Flow cytometry and WB are molecular approaches used to characterize the surface of the exosomes [35]. WB identified changes in the CD63, CD9, CD81, TSG101 and Alix surface proteins on the cancerous and premalignant exosomes. An increase in CD9 and CD81 and a decrease in CD63 proved to be markers of malignancy [25]. Recently, Gai et al. (2018) indicated that an increased presence of $\mathrm{CD} 44$ appears on the surface of salivary exosomes of cancer patients and is correlated with the changes of the miR-512-3p and 302b-3p expression [17].

\section{MicroRNAs in head and neck cancer}

The next step after exosome isolation and characterization is miR extraction from exosomal pellet (Fig. 2). In the papers reviewed, different kits were used for miR purification from each isolated exosome (e.g. Micro kit, Qiagen, Valencia, CA; TRIzol reagent, Invitrogen, Carlsbad, CA, USA; EXOs,
Zymoresearch Quick-gRNA ${ }^{\text {mo }}$ MiniPrep kit, USA; mirVana Isolation Kit, Thermo Fisher Scientific). The purified miRs were further quantified and confirmed using specific protocols and instruments (e.g. NanoDrop for quantification and Qubit fluorometer for confirmation). The main miR profiling methods are next-generation sequencing, quantitative realtime PCR (qRT-PCR) and miRNA microassay analysis [26]. The expression of the potential miR was quantified by droplet digital PCR (ddPCR) and qRT-PCR. qRT-PCR was preferred (4 articles) for this stage of analysis (Table 4).

miR dysregulation, as well as their presence or absence in tissues or biofluid samples, may represent an important diagnostic and prognostic factor for HNSCC. More specific, microRNAs not only reflect the changes induced by cancerous cells but may also represent the sophisticated instruments which sustain these changes. For example, in HNSCC, oncogenic miRs are upregulated, and they are responsible for targeting and silencing the tumor-suppressor genes which can modulate the outset, development and metastasis of cancerous cells. In contrast, tumor-suppressor miRs are downregulated, that reduce the modulation of oncogenes, sustaining the malignancy. Such an example is miR-200a reduction that facilitate epithelial-mesenchymal transition (EMT) by losing control over the modulation of $Z E B$ genes [19].

In the reviewed papers, salivary exosomal miR-10b-5p, miR-486-5p, miR-486-5-3p [16], miR-24-3p [18], miR134, miR-200a [19], miR-302b-3p, miR-517-3p, miR512-3p and miR-412-3p [17] had different levels of expressions in head and neck cancer saliva samples compared with normal (Table 4). Significantly higher expression of some of the above-mentioned miRs were proved by the authors, among which, miR-24-3p is 5.73fold elevated in cancer patients' saliva compared with healthy samples [18]. Farag et al. (2021) showed a significant downregulation of miR-200a in cancer samples [19]. This result was in agreement with Park et al.'s finding in cfmiR 200a [23]. Besides the overexpression of miR-412-3p and miR-512-3p in salivary extracellular vesicles of oral cancer patients, Gai et al. (2018) proved also that miR-302b-3p and miR-517b-3p were solely present in oral cavity SCC saliva samples [17]. Likewise, miR-4484, miR-1246 and miR-1290 were differently expressed in OLP saliva samples [15]. miR-4484 was significantly upregulated in salivary exosomes of OLP patients with a range between 2- and 98-fold [15].

Regarding sensitivity and specificity for head and neck cancer detection, the authors obtained various results. The highest specificity (100\%) was obtained by Langevin et al. (2017) by using miR-10b-5p, but it was associated with a very low sensitivity of $18 \%$ [16]. However, identification of both miR-10b-5p and miR-486-5p in saliva could discriminate cancer samples from healthy samples with an accuracy of $85 \%$ [16]. A more balanced result 
Table 4 MicroRNA types and mechanisms of action

\begin{tabular}{llll}
\hline Author & RNA analysis & microRNAs & Mechanisms of action \\
\hline Byun et al. (2015) [15] & miRNA-Ma, qRT-PCR & $\begin{array}{l}\text { miR-4484, } \\
\text { miR-1246,1290 } \\
\text { Langevin et al. (2017) [16] }\end{array}$ & miRN-10B-5p, \\
& & $\begin{array}{l}\text { miR-486-5p, } \\
\text { miR-486-3p }\end{array}$ & TLR, FCR \\
Gai et al. (2018) [17] & miR-302b-3p, miR-517-3p, miR-512-3p, miR-412-3p & ErbB, \\
& qRT-PCR assay, & & TGF Beta signaling pathways, \\
Ge et al. (2020) [18] & miRNA-MA, qRT-PCR & miR-24-3p & PER 1 \\
Farag et al. (2021) [19] & qRT-PCR & miR-134, & PDCD7, \\
& & miR-200a & EMT \\
\hline
\end{tabular}

between sensitivity and specificity was obtained by $\mathrm{He}$ et al. (2020), who showed a sensitivity and specificity of $64.4 \%$ and $80 \%$ respectively, by using miR-24-3p [18]. Gai et al. (2018) also proved a high sensitivity and specificity expressed by maximum Younden's index with AUC values of 0.847 and 0.871 for miR-512-3p and miR-412-3p respectively [17]. The statistical analysis was based on different cutpoints of miR expression. For example, miR-10b-5p had a cutpoint of $>1.0$ copies $/ \mu \mathrm{L}$, and miR-486-5p had a cutpoint of $>100$ copies/ $\mu \mathrm{L}$ [16].

Salivary exosomal miR detection presents some advantages over other possible HNC biomarker-based detection. Salivary exosomal miRs can be detected in small amounts of saliva, and they can be identified either by the analysis of the whole saliva or selectively the supernatant $[26,31,33]$. Additionally, some of the HNC biomarkers proved to be site specific for the same pathological types. For example, miR 200 group and miR-485-5p proved to be specific for oral cavity SCC, and miR-10b-5p was associated with oropharynx SCC $[16,30]$. More precisely, Lin et al. indicated the SCC of the tonsils as the specific site for miR-200 family [30].

Salivary exosomal miRs are correlated with the stage of the disease and the histopathological type and grade. While miR134 expression was higher in high-grade OSCC, miR-200a expression was higher in low-grade tumors [19]. miR-486-5p was able to detect stage I of cancer, supporting that the salivary exosomal miRs are promising biomarkers for early-stage cancer detection [16].

In precancerous lesions, exosomal microRNAs can be used as tools for identification of the progression towards the malignant phase. miR-4484 and miR-10b-5p are examples that mark the transformation of OLP and oral dysplasia to OSCC $[15,26,39]$. Moreover, miR-200a was associated with smoking-induced epigenetic changes, and its lower expression was correlated with a higher risk of oral cancer development [19]. Besides pointing the malign transformation, miR-200a was significantly increased 12 months after radiotherapy treatment [40]. All these results indicate that salivary exosomal miRs are potential biomarkers for head and neck cancer monitorization and treatment evaluation.

HPV infection status can also be identified by miRs. MiR-486-5p was detected in salivary exosomes from p16-positive oropharyngeal SCC patients and controls, and miR-100-5p was associated with HPV-negative oropharyngeal SCC [16, 41]. Other miRs, such as miR-9 family, can discriminate between HPV-positive and -negative statuses associated with HNSCC [18, 41-43].

Some disadvantages of miR-based cancer detection can be related to non-cancerous conditions, such as smoking, inflammation and aging process, which may alter the miR expression and further interfere with the cancer diagnosis process [44]. For example, miR-24-3p is differently expressed in elderly compared to young adults [45]. The lack of a standardized protocol will lead to a lower concordance between results and further to a lack of validation between laboratories [33]. miRs proved to have various degradation times into different fluids. For example, miR-124a has rapidly decreased in saliva samples (less than 10\% detectable level after $3 \mathrm{~min}$ ) compared with miR-191 (approximately 30\% detectable level after $30 \mathrm{~min}$ ) [23, 46].

Different expression of a specific miRs in various histopathological types represent another disadvantage. For example, pancreatic ductal adenocarcinoma and oral and oropharyngeal SCC showed altered expression with different values of miR-200b. The role of miR-200b may represent an explanation. Functioning as regulators of gene expression, same miR may influence different biological pathways of two distinct malignant processes [21, $28]$. Also, the presence of miR-20-5p in the exosomes from both head and neck and cervical HPV positive SCC, indicate that miR-20-5p is a marker of HPVassociated infection of SCC and the mechanism of carcinogenesis is similar [41, 43]. In addition, the fact that altered expression of one miR may offer various and totally different information is exemplified by miR-4484, which represents a biomarker for OLP and can also predict the lymph node metastasis of HSCC. The changes 
in the immune response induced by miR-4484 may justify the superposition of this miR activity in the two pathologies [17].

\section{MicroRNA pathways of carcinogenesis}

Salivary exosomal miRs detain various mechanisms of action which can sustain tumor microenvironment and thus, contribute to the proliferation of tumor cells (Table 4). miR-486-5p and miR-10b-5p are involved in the host's immune response through negative modulation of targets, such as tool-like receptors (TLR) and Fc receptors $[47,48]$. In OSCC, TLR favor tumor progression and chemotherapy resistance especially through the recruitment of suppressive regulatory $\mathrm{T}$ cells [49]. Another miR that induces drug resistance (Tamoxifen) is miR-24-3p, which can also cause a shortening of the cell cycle and an increase in the proliferation rate of tumor cells and increase the efficacy of cell colony forming through PER1 pathway [50]. By directly targeting 3'UTR of PER1, which is involved in cancer antiproliferative effect, miR-24-3p inhibits its expression and lock the cell cycle [51]. Thus, these mechanisms explain the correlation between increased expression of miR-24-3p and decreased PER1 expression in HNSCC [18]. However, the mechanism of action of 24-3p is still debated in scientific literature, on different types of cancers, such as lung or breast $[52,53]$.

In contrast with the above-mentioned miRs, elevated levels of miR-134 increase growth and migration of tumor cells by reducing the E-cadherin expression and, further, interacting with Programmed Cell Death 7 (P7DC7) in OSCC $[19,54]$. In addition, miR-134 inactivates the $W W O X$ tumor suppressor genes contributing repeatedly to carcinogenesis. These pathways explain the poor survival rate of patients with OSCC, which was associated with high expression of miR-134 [54]. On the other hand, Salazar et al.'s results indicated a lower expression of miR-134 in OSCC saliva samples [55]. This inadvertence may be caused not only by the different sample examination, cell culture and saliva, but also by the contrasting method of quantification. While in ShihYuan Peng et al.'s study, miR-134 expression was upregulated after a qRT-PCR analysis, in Salazar et al., research miR-134 was found downregulated in the microarray data set $[45,55]$.

Salivary miR-10b and miR-200a are remarkable examples of tumor invasion and metastasis biomarkers [19, $32,56]$. An increase in miR-10b expression is a marker of progression from premalignant lesion to OSCC. Due to miR-10b involvement in oncosuppression, elevated levels of miR-10b may represent protective actions taken by the normal cells against malign transformation [57]. Furthermore, this biomarker may allow to distinguish between progressing or non-progressing oral low-grade dysplasia to OSCC [39]. miR-200a favors tumor invasion, metastasis and resistance to cancer therapy. Being classified as a tumor-suppressor miR that preserves epithelial phenotype, miR-200a inhibits EMT, tumor invasion and metastasis generation [36, 58, 59]. Consequently, the downregulation of miR-200a permits EMT and negatively influences host response against tumor expansion, and thus, it can be used as a marker of malignant transformation and invasion [23, 60]. Additionally, miR-200a is involved in the DNA methylation induced by tobacco smoking, that leads to progressive accumulation of multiple genetic abnormalities and further to development of OSCC $[60,61]$. Moreover, miR200 family is involved in ZEB1 and ZEB2 modified expression and in lytic replication of Epstein-Barr virus (EBV) in B cells in the EBV pathophysiology [30, 58].

Compared to above-mentioned miRs, miR-512-3p and miR-27a-3p are involved in ErbB signaling, which promotes cell proliferation and tumor survival $[17,62]$. Additionally, they increase the expression of CD 44, which promotes ERK1/2 phosphorylation and cancer invasiveness [63]. miR-512-3p, miR-412-3p, miR-27a-3p, and miR-302b-3p are also involved in targeting genes of the TGF $\beta$ signaling pathway and Bmil, that promote cells formation, migration and metastasis [64, 65]. miR27a-3p overexpression reduces the control of EMT through $Y A P$ and $M C P H 1$ modification [66].

Exosomal miRs from saliva samples seem to differentiate patients with oral and oropharyngeal cancer and premalignant lesion from healthy subjects [15-19] even in early stages [67]. The technologies used for the detection of miRNAs required less time and lower costs compared with other biomarkers, such as proteins. Also, the fact that miRNAs have a high degree of specificity and can be associated with cancer morpho-functional changes, gives these RNA molecules the advantage of being used as markers not only for the detection, but also for monitoring the progression of the disease [68]. MicroRNAs present intricate and various mechanisms of action, which indicate the wide involvement in malignancy patterns and also make them a confident fingerprint of cancer.

The majority of cancer associated miRs are concentrated in saliva and serum exosomes [33, 69]. Exosomes represent a supplementary barrier against miR enzymatic degradation due to their lipidic double layer. Moreover, exosomal miR originates from an active process of carcinogenesis, which aims to control the environment for tumor expansion [70]. Contrarily, cfmiRs can be passively released by dead and desquamated cells and can consequently induce an increased rate of false negative results in cancer detection [23, 31, 71].

Exosomes are independent biomarkers of cancer due to the aforementioned cancer-related changes in concentration, size, shape and aspect, as well as various expression of surface proteins. Based on these modifications, 
exosomes can individually diagnose the histopathological grade of tumor and can represent a prognostic marker for metastasis due to exosomes roles in intercellular communication, tumor proliferation and cellular growth. Exosomal changes reverse after treatment [19, 26, 27, 38]. Nevertheless, alcohol consumption induces changes in exosome characteristics, fact to be mentioned and taken into consideration for exosome evaluation $[18,60]$.

Given the above, the additional step of exosome isolation increases the accuracy of exosomal miRs based HNC diagnosis. Ultracentrifugation proves to be more predictable and induces less interobserver differences compared with the other methods for exosomal isolation. TEM, NTA and WB are the most reliable methods for salivary exosome characterization and flow cytometry and negative staining can point out additional information.

Detection of exosomal miRs from saliva samples is non-invasive, comfortable for the patient and less sensitive for harvesting and transport compared with other biomarkers, such as mRNA. It also has a robust stability and resistance to degradation in body fluids [26]. Moreover, exosomes are found in abundance in saliva sample compared to other biological fluids and they can be isolated from small amounts of liquid [27, 46]. Given the above, saliva is a promising liquid biopsy for mass screening and rapid detection of head and neck cancer.

We think that this systematic review met the scope of identifying the most reliable exosomal microRNAs in saliva samples and bring forward the methods of analyzing these biomarkers for head and neck cancer detection, in order to help future research in choosing the proper saliva processing method and the specific exosomal miRs for head and neck squamous cell carcinoma identification.

\section{Conclusions}

Exosomal miR-10b-5p, miR-486-5p, miR-24-3p and miR-200a are the most promising biomarkers for oral and oropharyngeal cancer detection identified in saliva samples. Moreover, miR-486-5p can be detected from early stages. Salivary miRs purification from exosomes improves the diagnosis accuracy due to the increased concentration of miRs in exosomes and the supplementary protection that exosomes offer to the nucleic acids. Saliva is a proper sample for rapid and noninvasive cancer diagnosis and patient's follow-up.

\section{Abbreviations}

miRNA: MicroRNA; HNSCC: Head and neck squamous cell carcinoma; EMT: Epithelial-mesenchymal transition; EV: Extracellular vesicles; MVB: Multivesicular bodies; RISC: RNA-induced silencing complex; 3'UTR: 3' Untranslated region; QUEST: Quality Evaluation Scoring Tool; CTC: Circulant tumor cells; cfmiR: Cell-free miR; TEM: Transmission electron microscopy; NTA: Nanoparticle-tracking analysis; WB: Western blot; OSCC: Oral squamous cell carcinoma; OLP: Oral lichen planus; qRT-PCR: Real-time polymerase chain reaction; TLR: Tool-like receptors

Acknowledgements

None.

Authors' contributions

F.C.I. and O.C. analyzed the papers; F.C.I., H.R., A.J., and M.H. designed the research; F.C.I., H.R., O.C., A.J., R.C.R, M.M., M.C., and M.H. wrote part of the manuscript and reviewed the paper. The authors read and approved the final manuscript.

Funding

Not applicable.

Availability of data and materials

Not applicable.

\section{Declarations}

Ethics approval and consent to participate

No ethical approval or consent to participate was necessary since the data collected did not include information of personal identification.

Consent for publication

Not applicable.

\section{Competing interests}

The authors declare that they have no competing interests.

\section{Author details}

'Department of Oral Radiology, "Iuliu Hatieganu" University of Medicine and Pharmacy, 400033 Cluj-Napoca, Romania. ${ }^{2}$ Department of Oral and Cranio-Maxillofacial Surgery, "Iuliu Hatieganu" University of Medicine and Pharmacy, 400033 Cluj-Napoca, Romania. ${ }^{3}$ Faculty of Dental Medicine, "Iuliu Hatieganu" University of Medicine and Pharmacy, 400033 Cluj-Napoca, Romania. ${ }^{4}$ Research Center for Functional Genomics, Biomedicine and Translational Medicine, "Iuliu Hatieganu" University of Medicine and Pharmacy, 400337 Cluj-Napoca, Romania. ${ }^{5}$ Department of Otorhinolaryngology, "Iuliu Hatieganu" University of Medicine and Pharmacy, Cluj-Napoca 400000, Romania

Received: 17 May 2021 Accepted: 8 June 2021

Published online: 30 June 2021

References

1. Lydiatt WM, Patel SG, O'Sullivan B, Brandwein MS, Ridge JA, Migliacci JC, Loomis AM, Shah JP (2017) Head and neck cancers-major changes in the American Joint Committee on cancer eighth edition cancer staging manual. CA Cancer J Clin 67(2):122-137. https://doi.org/10.3322/caac.21389

2. Sung H, Ferlay J, Siegel RL, Laversanne M, Soerjomataram I, Jemal A et al (2021) Global cancer statistics 2020: GLOBOCAN estimates of incidence and mortality worldwide for 36 cancers in 185 countries. CA Cancer J Clin 71(3): 209-249

3. Cohen N, Fedewa S, Chen AY (2018) Epidemiology and demographics of the head and neck cancer population. Oral Maxillofac Surg Clin North Am 30(4):381-395. https://doi.org/10.1016/j.coms.2018.06.001

4. Guidi A, Codeca C, Ferrari D (2018) Chemotherapy and immunotherapy for recurrent and metastatic head and neck cancer: a systematic review. Med Oncol 35(3):37. https://doi.org/10.1007/s12032-018-1096-5

5. Marur S, Forastiere AA (2016) Head and neck squamous cell carcinoma: update on epidemiology, diagnosis, and treatment. Mayo Clin Proc 91(3): 386-396. https://doi.org/10.1016/j.mayocp.2015.12.017

6. Milman N, Ginini L, Gil Z (2019) Exosomes and their role in tumorigenesis and anticancer drug resistance. Drug Resist Updat 45:1-12. https://doi.org/1 0.1016/j.drup.2019.07.003

7. Chung IM, Rajakumar G, Venkidasamy B, Subramanian U, Thiruvengadam M (2020) Exosomes: Current use and future applications. Clin Chim Acta 500: 226-232. https://doi.org/10.1016/j.cca.2019.10.022

8. Khushman M, Bhardwaj A, Patel GK, Laurini JA, Roveda K, Tan MC et al (2017) Exosomal markers (CD63 and CD9) expression pattern using 
immunohistochemistry in resected malignant and nonmalignant pancreatic specimens. Pancreas 46(6):782-788. https://doi.org/10.1097/MPA. 0000000000000847

9. Mohr AM, Mott JL (2015) Overview of microRNA biology. Semin Liver Dis 35(1):3-11. https://doi.org/10.1055/s-0034-1397344

10. Berindan-Neagoe I, Calin GA (2014) Molecular pathways: microRNAs, cancer cells, and microenvironment. Clin Cancer Res 20(24):6247-6253. https://doi. org/10.1158/1078-0432.CCR-13-2500

11. Vu LT, Gong J, Pham TT, Kim Y, Le MTN (2020) microRNA exchange via extracellular vesicles in cancer. Cell Prolif 53(11):e12877

12. Wu L, Zheng K, Yan C, Pan X, Liu Y, Liu J, Wang F, Guo W, He X, Li J, Shen Y (2019) Genome-wide study of salivary microRNAs as potential noninvasive biomarkers for detection of nasopharyngeal carcinoma. BMC Cancer 19(1): 843. https://doi.org/10.1186/s12885-019-6037-y

13. Shamseer L, Moher D, Clarke M, Ghersi D, Liberati A, Petticrew M et al (2015) Preferred reporting items for systematic review and meta-analysis protocols (PRISMA-P) 2015: elaboration and explanation. BMJ 350:97647

14. Whiting PF, Rutjes AW, Westwood ME, Mallett S, Deeks JJ, Reitsma JB, Leeflang MM, Sterne JA, Bossuyt PM, QUADAS-2 Group (2011) QUADAS-2: a revised tool for the quality assessment of diagnostic accuracy studies. Ann Intern Med 155(8):529-536. https://doi.org/10.7326/0003-4819-155-8-20111 0180-00009

15. Byun JS, Hong SH, Choi JK, Jung JK, Lee HJ (2015) Diagnostic profiling of salivary exosomal microRNAs in oral lichen planus patients. Oral Dis 21(8): 987-993. https://doi.org/10.1111/odi.12374

16. Langevin S, Kuhnell D, Parry T, Biesiada J, Huang S, Wise-Draper T, Casper K, Zhang X, Medvedovic M, Kasper S (2017) Comprehensive microRNAsequencing of exosomes derived from head and neck carcinoma cells in vitro reveals common secretion profiles and potential utility as salivary biomarkers. Oncotarget 8(47):82459-82474. https://doi.org/10.18632/oncota rget.19614

17. Gai C, Camussi F, Broccoletti R, Gambino A, Cabras M, Molinaro L, Carossa S, Camussi G, Arduino PG (2018) Salivary extracellular vesicle-associated miRNAs as potential biomarkers in oral squamous cell carcinoma. BMC Cancer 18(1):439. https://doi.org/10.1186/s12885-018-4364-Z

18. He L, Ping F, Fan Z, Zhang C, Deng M, Cheng B, Xia J (2020) Salivary exosomal miR-24-3p serves as a potential detective biomarker for oral squamous cell carcinoma screening. Biomed Pharmacother 121:109553. https://doi.org/10.1016/j.biopha.2019.109553

19. Farag AF, Sabry D, Hassabou NF, Alaa EL-Din Y. (2021) MicroRNA-134/ microRNA-200a derived salivary exosomes are novel diagnostic biomarkers of oral squamous cell carcinoma. Egypt Dent J 67(1):367-377. https://doi. org/10.21608/edj.2020.47990.1317

20. Bantz KC, Meyer AF, Wittenberg NJ, Im H, Kurtulus O, Lee SH et al (2011) Recent progress in SERS biosensing. Phys Chem Chem Phys 13(24):1155111567. https://doi.org/10.1039/c0cp01841d

21. Lin X, Lin D, Ge X, Qiu S, Feng S, Chen R (2017) Noninvasive detection of nasopharyngeal carcinoma based on saliva proteins using surface-enhanced Raman spectroscopy. J Biomed Opt 22(10):1-6. https://doi.org/10.1117/1. JBO.22.10.105004

22. Lousada-Fernandez F, Rapado-Gonzalez O, Lopez-Cedrun JL, Lopez-Lopez R, Muinelo-Romay L, Suarez-Cunqueiro MM (2018) Liquid biopsy in oral cancer. Int J Mol Sci 19(6):1704. https://doi.org/10.3390/ijms19061704

23. Park NJ, Zhou H, Elashoff D, Henson BS, Kastratovic DA, Abemayor E, Wong DT (2009) Salivary microRNA: discovery, characterization, and clinical utility for oral cancer detection. Clin Cancer Res 15(17):5473-5477. https://doi. org/10.1158/1078-0432.CCR-09-0736

24. Kaczor-Urbanowicz KE, Martin Carreras-Presas C, Aro K, Tu M, Garcia-Godoy F, Wong DT (2017) Saliva diagnostics - current views and directions. Exp Biol Med (Maywood) 242(5):459-472. https://doi.org/10.1177/1535370216681550

25. Chiabotto G, Gai C, Deregibus MC, Camussi G (2019) Salivary extracellular vesicle-associated exRNA as cancer biomarker. Cancers (Basel) 11(7):891. https://doi.org/10.3390/cancers11070891

26. Yoshizawa JM, Wong DT (2013) Salivary microRNAs and oral cancer detection. Methods Mol Biol 936:313-324. https://doi.org/10.1007/978-1-62 703-083-0_24

27. Nonaka T, Wong DTW (2017) Saliva-exosomics in cancer: molecular characterization of cancer-derived exosomes in saliva. Enzymes 42:125-151. https://doi.org/10.1016/bs.enz.2017.08.002

28. Anjum A, Hosein M (2019) Diagnostic importance of saliva - an overview. J Pak Dent Assoc 28(03):129-135. https://doi.org/10.25301/JPDA.283.129
29. Connolly JM, Davies K, Kazakeviciute A, Wheatley AM, Dockery P, Keogh I, Olivo M (2016) Non-invasive and label-free detection of oral squamous cell carcinoma using saliva surface-enhanced Raman spectroscopy and multivariate analysis. Nanomedicine 12(6):1593-1601. https://doi.org/10.101 6/j.nano.2016.02.021

30. Lin Z, Swan K, Zhang X, Cao S, Brett Z, Drury S, Strong MJ, Fewell C, Puetter A, Wang $X$, Ferris MB, Sullivan DE, Li L, Flemington EK (2016) Secreted oral epithelial cell membrane vesicles induce Epstein-Barr Virus reactivation in latently infected B cells. J Virol 90(7):3469-3479. https://doi.org/10.1128/JVl. 02830-15

31. Rapado-Gonzalez O, Majem B, Muinelo-Romay L, Alvarez-Castro A, Santamaria A, Gil-Moreno A et al (2018) Human salivary microRNAs in cancer. J Cancer 9(4):638-649. https://doi.org/10.7150/jca.21180

32. Ribeiro IP, de Melo JB, Carreira IM (2019) Head and neck cancer: searching for genomic and epigenetic biomarkers in body fluids - the state of art. Mol Cytogenet 12(1):33. https://doi.org/10.1186/s13039-019-0447-z

33. Gallo A, Tandon M, Alevizos I, Illei GG (2012) The majority of microRNAs detectable in serum and saliva is concentrated in exosomes. PLoS One 7(3): e30679. https://doi.org/10.1371/journal.pone.0030679

34. Yu LL, Zhu J, Liu JX, Jiang F, Ni WK, Qu LS et al (2018) A comparison of traditional and novel methods for the separation of exosomes from human samples. Biomed Res Int 2018:3634563

35. Gurunathan S, Kang MH, Jeyaraj M, Qasim M, Kim JH (2019) Review of the isolation, characterization, biological function, and multifarious therapeutic approaches of exosomes. Cells 8(4):307. https://doi.org/10.3390/cells8040307

36. Patel GK, Khan MA, Zubair H, Srivastava SK, Khushman M, Singh S et al (2019) Comparative analysis of exosome isolation methods using culture supernatant for optimum yield, purity and downstream applications. Sci Rep 9(1):5335. https://doi.org/10.1038/s41598-019-41800-2

37. Sharma S, Gillespie BM, Palanisamy V, Gimzewski JK (2011) Quantitative nanostructural and single-molecule force spectroscopy biomolecular analysis of human-saliva-derived exosomes. Langmuir 27(23):14394-14400. https://doi.org/10.1021/la2038763

38. Blackwell RH, Foreman KE, Gupta GN (2017) The role of cancer-derived exosomes in tumorigenicity \& epithelial-to-mesenchymal transition. Cancers (Basel) 9(8):105. https://doi.org/10.3390/cancers9080105

39. Yang $Y$, Li YX, Yang $X$, Jiang L, Zhou ZJ, Zhu YQ (2013) Progress risk assessment of oral premalignant lesions with saliva miRNA analysis. BMC Cancer 13(1):129. https://doi.org/10.1186/1471-2407-13-129

40. Greither T, Vorwerk F, Kappler M, Bache M, Taubert H, Kuhnt T, Hey J, Eckert AW (2017) Salivary miR-93 and miR-200a as post-radiotherapy biomarkers in head and neck squamous cell carcinoma. Oncol Rep 38(2):1268-1275. https://doi.org/10.3892/or.2017.5764

41. Peacock B, Rigby A, Bradford J, Pink R, Hunter K, Lambert D, Hunt S (2018) Extracellular vesicle microRNA cargo is correlated with HPV status in oropharyngeal carcinoma. J Oral Pathol Med 47(10):954-963. https://doi. org/10.1111/jop.12781

42. Salazar-Ruales C, Arguello JV, Lopez-Cortes A, Cabrera-Andrade A, GarciaCardenas JM, Guevara-Ramirez P et al (2018) Salivary microRNAs for early detection of head and neck squamous cell carcinoma: a case-control study in the high altitude Mestizo Ecuadorian population. Biomed Res Int 2018: 9792730

43. Hui AB, Lin A, Xu W, Waldron L, Perez-Ordonez B, Weinreb I et al (2013) Potentially prognostic miRNAs in HPV-associated oropharyngeal carcinoma. Clin Cancer Res 19(8):2154-2162. https://doi.org/10.1158/1078-0432. CCR-12-3572

44. Takahashi K, Yokota S, Tatsumi N, Fukami T, Yokoi T, Nakajima M (2013) Cigarette smoking substantially alters plasma microRNA profiles in healthy subjects. Toxicol Appl Pharmacol 272(1):154-160. https://doi.org/10.1016/j. taap.2013.05.018

45. Machida T, Tomofuji T, Ekuni D, Maruyama T, Yoneda T, Kawabata Y, Mizuno H, Miyai H, Kunitomo M, Morita M (2015) MicroRNAs in salivary exosome as potential biomarkers of aging. Int J Mol Sci 16(9):21294-21309. https://doi. org/10.3390/ijms160921294

46. Falamas A, Rotaru H, Hedesiu M (2020) Surface-enhanced Raman spectroscopy (SERS) investigations of saliva for oral cancer diagnosis. Lasers Med Sci 35(6):1393-1401. https://doi.org/10.1007/s10103-020-02988-2

47. Rakoff-Nahoum S, Medzhitov R (2009) Toll-like receptors and cancer. Nat Rev Cancer 9(1):57-63. https://doi.org/10.1038/nrc2541

48. Furness AJ, Vargas FA, Peggs KS, Quezada SA (2014) Impact of tumour microenvironment and FC receptors on the activity of immunomodulatory 
antibodies. Trends Immunol 35(7):290-298. https://doi.org/10.1016/j.it.2014 05.002

49. Rich AM, Hussaini HM, Parachuru VP, Seymour GJ (2014) Toll-like receptors and cancer, particularly oral squamous cell carcinoma. Front Immunol 5:464

50. Sun X, Xiao D, Xu T, Yuan Y (2016) miRNA-24-3p promotes cell proliferation and regulates chemosensitivity in head and neck squamous cell carcinoma by targeting CHD5. Future Oncol 12(23):2701-2712. https://doi.org/10.2217/ fon-2016-0179

51. Sotak M, Sumova A, Pacha J (2014) Cross-talk between the circadian clock and the cell cycle in cancer. Ann Med 46(4):221-232. https://doi.org/10.31 09/07853890.2014.892296

52. Yan L, Ma J, Zhu Y, Zan J, Wang Z, Ling L, Li Q, Lv J, Qi S, Cao Y, Liu Y, Cao L, Zhang Y, Qi Z, Nie L (2018) miR-24-3p promotes cell migration and proliferation in lung cancer by targeting SOX7. J Cell Biochem 119(5):39893998. https://doi.org/10.1002/jcb.26553

53. Khodadadi-Jamayran A, Akgol-Oksuz B, Afanasyeva Y, Heguy A, Thompson M, Ray K, Giro-Perafita A, Sánchez I, Wu X, Tripathy D, Zeleniuch-Jacquotte A, Tsirigos A, Esteva FJ (2018) Prognostic role of elevated mir-24-3p in breast cancer and its association with the metastatic process. Oncotarget 9(16):12868-12878. https://doi.org/10.18632/oncotarget.24403

54. Peng SY, Tu HF, Yang CC, Wu CH, Liu CJ, Chang KW, Lin SC (2018) miR-134 targets PDCD7 to reduce E-cadherin expression and enhance oral cancer progression. Int J Cancer 143(11):2892-2904. https://doi. org/10.1002/ijc.31638

55. Salazar C, Nagadia R, Pandit P, Cooper-White J, Banerjee N, Dimitrova N, Coman WB, Punyadeera C (2014) A novel saliva-based microRNA biomarker panel to detect head and neck cancers. Cell Oncol (Dordr) 37(5):331-338. https://doi.org/10.1007/s13402-014-0188-2

56. John K, Wu J, Lee BW, Farah CS (2013) MicroRNAs in head and neck cancer. Int J Dent 2013:650218

57. Cristaldi M, Mauceri R, Di Fede O, Giuliana G, Campisi G, Panzarella V (2019) Salivary biomarkers for oral squamous cell carcinoma diagnosis and followup: current status and perspectives. Front Physiol 10:1476. https://doi.org/1 0.3389/fphys.2019.01476

58. Arunkumar G, Deva Magendhra Rao AK, Manikandan M, Prasanna Srinivasa Rao H, Subbiah S, llangovan R, Murugan AK, Munirajan AK (2018) Dysregulation of miR-200 family microRNAs and epithelial-mesenchymal transition markers in oral squamous cell carcinoma. Oncol Lett 15(1):649657. https://doi.org/10.3892/ol.2017.7296

59. Tellez CS, Juri DE, Do K, Bernauer AM, Thomas CL, Damiani LA, Tessema M, Leng S, Belinsky SA (2011) EMT and stem cell-like properties associated with miR-205 and miR-200 epigenetic silencing are early manifestations during carcinogen-induced transformation of human lung epithelial cells. Cancer Res 71(8):3087-3097. https://doi.org/10.1158/0008-5472.CAN-10-3035

60. Momen-Heravi F, Bala S, Kodys K, Szabo G (2015) Exosomes derived from alcohol-treated hepatocytes horizontally transfer liver specific miRNA-122 and sensitize monocytes to LPS. Sci Rep 5(1):9991. https://doi.org/10.1038/ srep09991

61. Wiklund ED, Gao S, Hulf T, Sibbritt T, Nair S, Costea DE, Villadsen SB, Bakholdt V, Bramsen JB, Sørensen JA, Krogdahl A, Clark SJ, Kjems J (2011) MicroRNA alterations and associated aberrant DNA methylation patterns across multiple sample types in oral squamous cell carcinoma. PLoS One 6(11):e27840. https://doi.org/10.1371/journal.pone.0027840

62. Appert-Collin A, Hubert P, Cremel G, Bennasroune A (2015) Role of ErbB receptors in cancer cell migration and invasion. Front Pharmacol 6:283

63. Judd NP, Winkler AE, Murillo-Sauca O, Brotman JJ, Law JH, Lewis JS Jr, Dunn GP, Bui JD, Sunwoo JB, Uppaluri R (2012) ERK1/2 regulation of CD44 modulates oral cancer aggressiveness. Cancer Res 72(1):365-374. https://doi. org/10.1158/0008-5472.CAN-11-1831

64. Meng W, Xia Q, Wu L, Chen S, He X, Zhang L, Gao Q, Zhou H (2011) Downregulation of TGF-beta receptor types II and III in oral squamous cell carcinoma and oral carcinoma-associated fibroblasts. BMC Cancer 11(1):88. https://doi.org/10.1186/1471-2407-11-88

65. Liu W, Feng JQ, Shen XM, Wang HY, Liu Y, Zhou ZT (2013) Two stem cell markers, ATP-binding cassette, G2 subfamily (ABCG2) and BMI-1, predict the transformation of oral leukoplakia to cancer: a long-term follow-up study. Cancer 118(6):1693-1700

66. Venkatesh T, Nagashri MN, Swamy SS, Mohiyuddin SM, Gopinath KS, Kumar A (2013) Primary microcephaly gene MCPH1 shows signatures of tumor suppressors and is regulated by miR-27a in oral squamous cell carcinoma. PLoS One 8(3):e54643. https://doi.org/10.1371/journal.pone.0054643
67. Carofino BL, Dinshaw KM, Ho PY, Cataisson C, Michalowski AM, Ryscavage A Alkhas A, Wong NW, Koparde V, Yuspa SH (2019) Head and neck squamous cancer progression is marked by CLIC4 attenuation in tumor epithelium and reciprocal stromal upregulation of miR-142-3p, a novel post-transcriptional regulator of CLIC4. Oncotarget 10(68):7251-7275. https://doi.org/10.18632/ oncotarget.27387

68. Condrat CE, Thompson DC, Barbu MG, Bugnar OL, Boboc A, Cretoiu D et al (2020) miRNAs as biomarkers in disease: latest findings regarding their role in diagnosis and prognosis. Cells 9(2):276

69. Arroyo JD, Chevillet JR, Kroh EM, Ruf IK, Pritchard CC, Gibson DF, Mitchell PS, Bennett CF, Pogosova-Agadjanyan EL, Stirewalt DL, Tait JF, Tewari M (2011) Argonaute2 complexes carry a population of circulating microRNAs independent of vesicles in human plasma. Proc Natl Acad Sci U S A 108(12): 5003-5008. https://doi.org/10.1073/pnas.1019055108

70. Deregibus MC, Figliolini F, D'Antico S, Manzini PM, Pasquino C, De Lena M et al (2016) Charge-based precipitation of extracellular vesicles. Int J Mol Med 38(5):1359-1366. https://doi.org/10.3892/ijmm.2016.2759

71. Nowicka Z, Stawiski K, Tomasik B, Fendler W (2019) Extracellular miRNAs as biomarkers of head and neck cancer progression and metastasis. Int J Mol Sci 20(19):4799. https://doi.org/10.3390/ijms20194799

\section{Publisher's Note}

Springer Nature remains neutral with regard to jurisdictional claims in published maps and institutional affiliations.

\section{Submit your manuscript to a SpringerOpen ${ }^{\circ}$ journal and benefit from:}

- Convenient online submission

- Rigorous peer review

- Open access: articles freely available online

- High visibility within the field

- Retaining the copyright to your article

Submit your next manuscript at $\boldsymbol{\nabla}$ springeropen.com 